\title{
GRAVITY EFFECTS IN THE WATER ENTRY PROBLEM
}

\author{
A. G. MACKIE
}

(Received 23 November 1964)

\section{Introduction}

The following paper is a sequel to the author's earlier paper [2]. In that paper some general results were obtained which described the motion of a fluid with a free surface subsequent to a given initial state and prescribed boundary conditions of a certain type. The analysis was based on a linearized theory but gravity effects were included. Viscosity, compressibility and surface tension effects were neglected. Among the problems treated was that of the normal symmetric entry of a thin wedge into water at rest. This water entry problem has attracted a considerable amount of attention since the pioneer paper by Wagner [5]. Both linear and non-linear approximations have been used but all papers apart from [2] neglect gravity on the assumption that in the early stages of the penetration this is unimportant. One of the objects of [2] was to determine the solution with the gravity terms retained. A formal solution was obtained but no attempt was made to analyse this quantitatively. In the present paper we examine the extent of this effect in some detail. It will be of help to the reader to have some familiarity with the first three or four sections of [2] but in order to make the present paper self-contained we shall first reintroduce the notation used there and quote the necessary results from that paper without proof.

We consider an incompressible fluid with a free surface whose equation is $y=\eta(x, t)$. The plane $y=0$ is the mean free surface, $\eta$ is assumed small and the $y$-axis points vertically downwards. In the first instance twodimensional problems only are considered and symmetry about the $y$-axis is also assumed. Although two-dimensional problems are less important than those with axial symmetry, this specialization is made in the interests of mathematical simplicity and the extension to axially symmetric flow will be made where feasible. The fluid is non-viscous and the flow assumed to be irrotational. Accordingly there exists a velocity potential $\phi(x, y, t)$ which is harmonic in the variables $x$ and $y$. On $y=0, \phi$ satisfies the boundary condition

$$
\frac{\partial^{2} \phi}{\partial t^{2}}-g \frac{\partial \phi}{\partial y}=0 \text {. }
$$


We denote by bars the Fourier cosine transform with respect to $x$ so that, in particular,

$$
\bar{\eta}(\lambda, t)=\int_{0}^{\infty} \eta(x, t) \cos \lambda x d x
$$

The main result of [2] is the determination of the motion of the fluid, at rest for $t<0$, when a wavemaker acts along the boundary $x=0, y>0$ in such a way as to supply the boundary condition

It is found that

$$
\frac{\partial \phi}{\partial x}=U(y, t) \quad(x=0, y \geqq 0, t \geqq 0) .
$$

$$
\bar{\eta}(\lambda, t)=-\int_{0}^{t} \tilde{U}(\lambda, \tau) \cos \left\{(\lambda g)^{\frac{1}{2}}(t-\tau)\right\} d \tau,
$$

where

$$
\tilde{U}(\lambda, t)=\int_{0}^{\infty} U(y, t) e^{-\lambda y} d y,
$$

and from these equations the equation of the free surface $\eta(x, t)$ is recovered by Fourier inversion. We consider in particular a thin symmetric body which rests when $t<0$ with its tip at the origin and its axis vertical. Its shape in this position is given by $x=f(y)$, where $f$ is small in some sense, is itself positive but is defined for negative values of its argument only. If this body is plunged at $t=0$ with constant speed $U$ into the water,

$$
U(y, t)=\left\{\begin{array}{cl}
-U f^{\prime}(y-U t) & (x=0,0 \leqq y \leqq U t) \\
0 & (x=0, y>U t)
\end{array}\right.
$$

For a thin wedge, $f(y)=-\varepsilon y$, where $\varepsilon$ is small, and after some algebra we find the equation of the free surface to be

$$
\eta(x, t)=\frac{2 \varepsilon U^{2}}{\pi} \int_{0}^{\infty} \frac{\cos \lambda x}{\lambda\left(\lambda U^{2}+g\right)}\left\{\cos (\lambda g)^{\frac{1}{2}} t-U(\lambda / g)^{\frac{1}{2}} \sin (\lambda g)^{\frac{1}{t}} t-e^{-\lambda U t}\right\} d \lambda .
$$

\section{Investigation of the splash profile}

The splash profile is not by itself the most important physical characteristic of the water entry problem but it is one which is easily visualized and also one which admits of comparatively detailed analysis. Moreover, one would hope to get some general indication of gravity effects by means of such an investigation. The usual arguments concerning the "early stages" of the entry refer to times $t$ which are small compared with $U / g$, the only combination of the data which has the dimension of time. We therefore seek the asymptotic form of $\eta$ as given by (5). We can do this in two ways. We 
can try to find the displacement at a given position for small $t$. We can also seek the displacement at a given time for large values of $x$.

The second of these problems is more straightforward and it will be discussed first. Examination of the integrand in (5) shows it to be of the form $\cos \lambda x F(\lambda)$, where $F(\lambda)$ is an analytic function of $\lambda$ whose only singularity is a simple pole at $\lambda=-g / U^{2}$ and which is of order $\lambda-\frac{3}{2}$ for large real positive values of $\lambda$. It follows that an asymptotic expansion for large values of $\boldsymbol{x}$ can be obtained by integration by parts, leading to the form

$$
\eta(x, t)=-\frac{2 \varepsilon U^{2}}{\pi}\left(\frac{F^{\prime}(0)}{x^{2}}-\frac{F^{\prime \prime \prime}(0)}{x^{4}}+\cdots\right) .
$$

After some manipulation, this produces the expansion

$$
\begin{aligned}
\frac{\eta}{x}= & -\frac{\varepsilon}{\pi}\left[\frac{1}{3}\left(\frac{U t}{x}\right)^{3}\left(1+\frac{g t}{4 U}\right)\right. \\
& \left.-\frac{1}{10}\left(\frac{U t}{x}\right)^{5}\left\{1+\frac{g t}{6 U}+\frac{1}{42}\left(\frac{g t}{U}\right)^{2}+\frac{1}{336}\left(\frac{g t}{U}\right)^{3}\right\}\right]+o\left(\frac{1}{x^{7}}\right) .
\end{aligned}
$$

When $g=0$, the expression for $\eta(x, t)$ can be found explicitly, either by letting $g \rightarrow 0$ in (5) and evaluating the appropriate integral or, more directly, by solving the boundary value problem as in [1] with $\phi=0$ as a boundary condition on $y=0$ instead of (1). If the value of $\eta$ when $g=0$ is denoted by $\eta_{0}$, either of these methods gives

$$
\frac{\eta_{0}}{x}=\frac{\varepsilon}{\pi}\left\{\frac{2 U t}{x}-2 \tan ^{-1} \frac{U t}{x}-\frac{U t}{x} \log \left(1+\frac{U^{2} t^{2}}{x^{2}}\right)\right\} .
$$

It is easily verified that the terms in (7) which are not $O\left(x^{-7}\right)$ coincide with those obtained by setting $g=0$ in (6).

We can now examine the nature and magnitude of the effects of gravity terms in the equation of the splash profiele. We note that for large $x, \eta$ is negative as expected since $y$ is measured vertically downwards but that the effect of the gravity term is to increase the displacement away from the undisturbed free surface. At first sight this seems a curious result since one would intuitively expect the free surface to be kept down further by the action of gravity forces. However, we must remember that there is built into our system of equations the principle of conservation of volume and that the volume of liquid displaced in a given time is the same whether gravity terms are present or not. Thus the area under the $\eta$ curve is independent of $g$ and we see that the presence of gravity terms tends to depress the splash profile near the body but to introduce a compensating increase further away. This point is emphasized by the further observation that $\eta_{0}$, as given by (7), has a logarithmic singularity at $x=0$ whereas $\eta$, as given by (5) 
is finite at $x=0$ since the integral in (5) is absolutely and uniformly convergent for $x \geqq 0$. This shows that the $\eta$ and $\eta_{0}$ curves must cross over at some point. The general shape of the $\eta_{0}$ curve can be easily determined. It changes monotonically from its logarithmic infinity at $x=0$ to a zero of order $x^{-2}$ at $x=\infty$. The $\eta$ curve, on the other hand, is finite at $x=0$ and for large $x$ at a given time $t$

$$
\eta(x, t)=\eta_{0}(x, t)\left(1+\frac{g t}{4}\right)+o\left(\frac{1}{x^{4}}\right) .
$$

An additional point of contrast is that the $\eta$ curve will have a sinusoidal profile and, away from $x=0$, will be not unlike the surface profile occurring in the Cauchy-Poisson initial value problem (see, for example [3] pp. $156-163)$.

It is possible to obtain the leading terms in the expansion of the splash profile for large $x$ when an arbitrary thin body, whose shape at $t=0$ is $x=f(y)$, enters the water with constant speed $U$. From (2), (3) and (4)

$$
\bar{\eta}(\lambda, t)=U \int_{0}^{t} \cos \left\{(\lambda g)^{\frac{1}{2}}(t-\tau)\right\} d \tau \int_{0}^{U \tau} f^{\prime}(\alpha-U \tau) e^{-\lambda \alpha} d \alpha .
$$

The function $\eta(x, t)$ is found from $\bar{\eta}$ by Fourier inversion and, after integration by parts,

$$
\eta(x, t)=-\frac{2 \bar{\eta}^{\prime}(0, t)}{\pi x^{2}}+O\left(\frac{1}{x^{4}}\right),
$$

where the prime denotes differentiation with respect to $\lambda$. If (8) is differentiated with respect to $\lambda, \lambda$ is set equal to zero and the orders of integration interchanged, we obtain after some manipulation

(9) $\eta(x, t)=-\frac{2}{\pi x^{2}}\left\{\int_{0}^{U t} \alpha f(\alpha-U t) d \alpha+\frac{U g}{2} \int_{0}^{t}(t-\tau)^{2} f(-U \tau) d \tau\right\}+o\left(\frac{1}{x^{2}}\right)$.

This shows that, for a thin body of arbitrary shape, the effect of gravity is always to increase the height of the splash profile far away from the body. The appropriate part of the earlier result (6) can be obtained by setting $f(y)=-\varepsilon y$ in $(9)$.

The compensating depression nearer to the origin is most clearly seen by an example which is not exactly a water entry problem but is closely akin to one. If an explosion is set off in the water at $t=0$ at the point $x=0, y=Y$, then we may obtain some representation of this by setting $U(y, t)=\delta(y-Y) \delta(t)$, where $\delta$ denotes the Dirac delta function. By using the techniques derived in [2] we obtain

$$
\eta(x, t)=-\frac{2}{\pi} \int_{0}^{\infty} e^{-\lambda Y} \cos \left\{(\lambda g)^{\frac{1}{2}} t\right\} \cos \lambda x d \lambda .
$$


When $g=0$, the splash profile can be evaluated explicitly and if we denote this by the suffix zero as before, then

$$
\eta_{0}(x, t)=-\frac{2 Y}{\pi\left(x^{2}+Y^{2}\right)} .
$$

In this idealized model, $\eta_{0}$ is independent of $t$ and the splash profile is formed instantaneously and permanently. However, when $g \neq 0$, integration by parts gives

$$
\eta(x, t)=-\frac{2}{\pi x^{2}}\left(Y+\frac{g t^{2}}{2}\right)+O\left(\frac{1}{x^{4}}\right),
$$

showing the characteristic behaviour for large $x$. On the other hand, when $x=0$ it is clear from (10) that $|\eta|<\left|\eta_{0}\right|$, since the presence of the oscillating cosine term coming from the fact that $g \neq 0$ must decrease the absolute value of the integrand. The delta function explosion model is similar to the water entry problem in this respect that a fixed amount of fluid is released to cause the splash profile and that this amount is independent of $g$. The integrated curves of $\eta$ and $\eta_{0}$ over all $x$ must therefore be the same.

We return now to the wedge problem and examine the more delicate asymptotic solution of (5) for a given position $x$ and small values of the time $t$. We note that it is impossible to expand the integrand in powers of $t$ since this immediately causes the integrals with respect to $\lambda$ to diverge. However, the expansion of $\eta$ in powers of $t$ for fixed $x$ can be accomplished by an ingenious device suggested in a letter to the author by Professor E. T. Copson to whom he is greatly indebted. This consists of forming the quantity $U \ddot{\eta}-g \dot{\eta}$, where the dots denote differentiation with respect to $t$. This leads to the equation

$$
\begin{aligned}
U \dot{\eta}-g \dot{\eta}= & -\frac{2 \varepsilon U^{4} t}{\pi\left(x^{2}+U^{2} t^{2}\right)} \\
& +2 \varepsilon U^{2}\left(\frac{2 g}{\pi x}\right)^{\frac{1}{2}}\left\{C\left(\frac{g t^{2}}{4 x}\right) \sin \frac{g t^{2}}{4 x}-S\left(\frac{g t^{2}}{4 x}\right) \cos \frac{g t^{2}}{4 x}\right\},
\end{aligned}
$$

where $C, S$ are forms of Fresnel integrals defined by

$$
C(\theta)=\frac{1}{(2 \pi)^{\frac{1}{2}}} \int_{0}^{\theta} z^{-\frac{1}{2}} \cos z d z, \quad S(\theta)=\frac{1}{(2 \pi)^{\frac{1}{2}}} \int_{0}^{\theta} z^{-\frac{1}{2}} \sin z d z .
$$

By this device the expression for $\eta$ is transformed from the evaluation of a somewhat complicated Fourier integral to the solution of an ordinary, second order, non-homogeneous differential equation with constant coefficients and initial conditions $\eta=\dot{\eta}=0$. The complete solution can easily be written down in terms of the appropriate Green's function but for our purposes 
we shall merely obtain the leading terms in the expansion of $\eta$ for fixed $x$ and small $t$. Up to and including terms in $t^{6}$ this expansion is

$$
\frac{\eta}{x}=-\frac{\varepsilon}{3 \pi}\left(\frac{U t}{x}\right)^{3}\left(1+\frac{g t}{4 U}\right)+\frac{\varepsilon}{10 \pi}\left(\frac{U t}{x}\right)^{5}\left(1+\frac{g t}{6 U}\right)+O\left(t^{7}\right) .
$$

In this expression, contributions from the terms in (11) which involve Fresnel integrals enter only in the terms of order $t^{5}$ and higher. The $g$ which arises in the term in $t^{4}$ on the right hand side of (12) comes exclusively from the existence of the term $-g \dot{\eta}$ in the left hand side of (11).

\section{The case of axial symmetry}

When a thin axially symmetric body is thrust with constant speed $U$ into the water, it was shown in [2] that substantially similar methods could be used to determine the splash profile as were used for the two-dimensional case. The main difference was the replacement of Fourier cosine transforms with respect to $x$ by Hankel transforms of order zero with respect to $r$, the radial co-ordinate. Application of these methods gives the equation of the splash profile when a thin cone of apex angle $2 \varepsilon$ is thrust normally into the water with constant speed $U$ as

$$
\begin{aligned}
& \eta(r, t)=\frac{\varepsilon^{2} U^{2}}{g} \int_{0}^{\infty} \frac{J_{0}(\lambda r)}{\lambda\left(\lambda U^{2}+g\right)}
\end{aligned}
$$

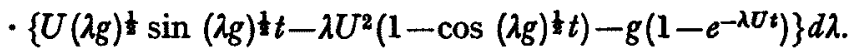

Using the ideas of the previous section we determine $U \dot{\eta}-g \eta$ and obtain

$$
U \dot{\eta}-g \eta=\varepsilon^{2} U^{2} \int_{0}^{\infty} \frac{J_{0}(\lambda r)}{\lambda}\left\{1-e^{-\lambda U t}-U(\lambda / g)^{\frac{1}{2}} \sin (\lambda g)^{\frac{1}{2}} t\right\} d \lambda .
$$

Evaluation of the last integral in this expression presents some difficulty but a series form has been given by Terazawa [4] and using this we can write

$$
U \dot{\eta}-g \eta=\varepsilon^{2} U^{2}\left\{\sinh ^{-1} \frac{U t}{r}-\frac{U t}{r} \sum_{n=0}^{\infty} \frac{(-1)^{n} n !}{(2 n+1) !}\left(\frac{g t^{2}}{r}\right)^{n} P_{n}(0)\right\} .
$$

The solution of this equation for which $\eta=0$ when $t=0$ is

$$
\eta(r, t)=-\frac{\varepsilon^{2} U^{4} t^{4}}{24 r^{3}}\left(1+\frac{g t}{5 U}\right)+\cdots
$$

where careful examination shows that the missing terms are $o\left(t^{5}\right)$ for fixed $r$ and small $t$ but are also $o\left(r^{-3}\right)$ for fixed $t$ and large $r$. It is observed that the general pattern of behaviour is much as in the two-dimensional case but with gravity effects being slightly less significant after the same interval of time. 


\section{Conclusions}

The splash profile for the water entry problem has been examined on the basis of a linearized theory which includes gravity effects. Justification for neglecting gravity in previous papers rested on the assumption that such effects would be unimportant for values of $t \ll U / g$. A critique for this assertion is established by the demonstration that, to a first approximation, the splash profile is affected by a factor of magnitude $1+g t / 4 U$ and $1+g t / 5 U$ in the two-dimensional and (axially symmetric) three-dimensional cases respectively. Further, it is shown that gravity tends to decrease the height of the splash profile near to the body and to increase it further away. The inclusion of gravity effects, besides being more realistic physically, has the satisfactory mathematical consequence that the splash profile is now uniformly bounded instead of possessing a singularity at the origin.

We have made no mention of drag forces on the body. It is possible to obtain an expression for the drag force for the entry of a wedge when $g=0$ by integrating the contribution from the unsteady term $\partial \phi / \partial t$ in Bernoulli's equation. The resultant vertical drag is of order $\varepsilon^{2}$. When gravity terms are present the hydrostatic pressure on the wedge is of order unity and when this force is resolved vertically it is of order $\varepsilon$. There is thus no basis for comparison. We may therefore assume that, for thin bodies, the ordinary hydrostatic pressure is dominant in offering resistance to the progress of the body into the water.

\section{References}

[1] Mackie, A.G., A linearized theory of the water entry problem, Quart. J. Mech. Appl. Math. 15 (1962), $137-151$.

[2] Mackie, A. G., Initial value problems in water wave theory, J. Austral. Math. Soc. 3 (1963), $340-350$.

[3] Stoker, J. J., Water Waves, (Interscience, New York, 1957).

[4] Terazawa, K., On deep-sea water waves caused by a local disturbance on or beneath the surface, Proc. Roy. Soc. A 92 (1916), 57-81.

[5] Wagner, H., Uber Stoss- und Gleitvorgänge an den Oberflächen von Flüssigkeiten, $Z$. Angew. Math. Mech. 12 (1932), 193-215.

Victoria University of Wellington

Wellington, New Zealand. 